\title{
Perspectives in preschool nutrition
}

\author{
Preethi Rahul $^{1}$, Amit Khandeparkar ${ }^{2}$ \\ From ${ }^{1}$ Assistant Manager, ${ }^{2} \mathrm{Head}$, Department of Healthcare Nutrition Science, Nutricia International Pvt. Ltd. (Danone India), Mumbai, Maharashtra, \\ India
}

\begin{abstract}
Malnutrition is a grave global public health problem and needs serious attention. The prevalence of malnutrition in under 5 years children in India is high. Hence, there is an urgent need to recognize the nutrition and feeding concerns, the gaps in adequate nutrient intake and bridge them to ensure optimum nutritional care in early childhood. Twenty-eight Pediatricians were selected by purposive sampling from Telangana $(\mathrm{n}=12)$ and West Bengal $(\mathrm{n}=16)$ to form an advisory board. An advisory board meeting was conducted, which included a presentation on preschool nutrition in an Indian perspective. This was followed by a focused group discussion among the team of experts to gather their views on nutrition concerns of the preschoolers. They gave their opinion on how to address these concerns, role of healthy food drinks, the role of novel nutrients such as prebiotics and docosahexaenoic acid in preschoolers and the importance of nutrition education of mothers by healthcare professionals. Further, the board members pointed out the common micronutrient deficiencies such as iron, Vitamins-A and D, excessive intake of sugar and fat, and indicated a need for micronutrient supplementation, healthy alternatives for sugar and fat, views on healthy food drinks among the preschoolers. The advisory board members were unanimous in their belief that it is essential to create awareness and educate mothers about preschool nutrition and for doctors to include some nutrition counseling time for early childhood nutritional care. This would help to improve the nutritional intake which is essential to ensure optimum growth and development during the preschool age for a healthier future.
\end{abstract}

Key words: Child nutrition, Malnutrition, Micronutrient deficiencies, Pediatrics, Preschooler, Under 5 years

$\mathrm{T}$ The World Health Organization (WHO) reported malnutrition as a world health crisis in 2019 [1]. In India, the recent National Family and Health Survey 5 (2019-2020), confirmed the high prevalence of malnutrition in under 5 years age, indicating a need for the management of the triple burden (undernutrition, over nutrition, and micronutrient deficiencies) of early childhood malnutrition [2]. Preschool years are crucial years for growth and development and balanced dietary intake is an important factor for healthy growth and development during childhood.

\section{Preschooler - Definition and Characteristics}

A child in the age group of 3-5 years is defined as a preschooler by the Centre of Disease Control and prevention [3]. This is a critical phase in every child's life as this is the time when the child grows, develops linguistic skills, and attains an increased sense of independence [4]. During preschool years, the environment stimulating the child's brain as it matures, brings with it the characteristic traits such as egoistic behavior, lack of true consideration, bouts of frustration, aggression,

\section{Access this article online}

Received - 02 November 2021

Initial Review - 10 November 2021

Accepted - 15 November 2021

DOI: $10.32677 /$ ijch.v8i11.3137 playfulness, social shyness, closeness, contact and comfort from parents, fear of separation, lack of self-control, and impulsiveness. These behavioral patterns determine their personality. They also attain their age related sensory and motor developmental milestones during this period [5].

Factors Impacting Growth and Development of Preschoolers

Nurturing children during these years is crucial to ensure a healthy physical growth and attaining the developmental milestones. Following are the main factors impacting childhood growth and development [6]:

- Parenting styles and behavior: Parenting styles and behavior have an influence on developing the life-long health habits [7]

- Socio-cultural factors: Social customs and norms in the communities, family members and caregivers can influence the child's eating habits, impacting their growth [8]

- Environmental factors: UNICEF 2019 review reflected that easy access to safe drinking water and improved individual household sanitation can reduce the infection risk, malabsorption, malnutrition and mortality [9]

Correspondence to: Preethi Rahul, Nutricia International Private Limited, Unit No.304, $3^{\text {rd }}$ Floor, Building A, Piramal Agastya Corporate Park, LBS Road, Kurla, Mumbai - 400 070, Maharashtra, India. E-mail: preethi.rahul@danone.com

(C) 2021 Creative Commons Attribution-NonCommercial 4.0 International License (CC BY-NC-ND 4.0). 
- Maternal and Child nutrition: UNICEF reported in 2018 that improvements in maternal and child nutrition, and healthy environment could improve child's nutritional status, growth and development [9].

\section{Food Consumption Patterns in Indian Preschool Children}

As per the findings of the Comprehensive National Nutrition Survey (CNNS) Report 2016-2018, dietary intake of 2-4 years old children in India is represented in Figure 1. The consumption patterns were similar among both genders. Urban families with higher income showed greater dietary diversity. Dairy products were less frequently consumed with only two-thirds of schoolage children and adolescents consuming milk or curd at least once per week while fruits and non-vegetarian foods were consumed least frequently [10]. These food-based patterns reveal that the preschoolers are at a higher risk to develop deficiencies of protein, docosahexaenoic acid (DHA), vitamins A and D, iron and calcium and dietary fibre.

\section{Key nutritional Concerns of Preschool Children}

\section{Dental caries}

Increased intake of sugar rich foods has increased the risk of dental caries and poses a serious threat to oral health. Research indicated that, in 3-6-year-old children, caries free children scored better on healthy eating index scores and scores of solid fat, alcohol and added sugars (SoFAAS) compared to their counterparts, signifying its lower intake which is in agreement with the cariogenic role of sugars seen in numerous studies [11].

\section{Iron deficiency and anemia}

The WHO (2021) has estimated iron deficiency anemia in $42 \%$ of preschoolers [12]. According to the CNNS report 2016-2018, $41 \%$ of preschoolers and $24 \%$ of school-going children were anemic. Anemia was a moderate or severe public health problem among preschoolers in 27 states. Prevalence of anemia as a public health problem was found to be moderate in Telangana and severe in Kolkata. Overall, $32 \%$ of preschoolers and $17 \%$ of school-going children had iron deficiency (low serum ferritin) in India [10].

\section{Constipation}

Constipation in Indian children is becoming a common complaint and most of it is functional (95\%). Post 2000, the increasing sedentary life has led to a rise in the prevalence of functional constipation in preschoolers [13].

\section{Diarrhea}

Despite measures being taken in controlling diarrhoea related mortality, the burden of the disease remains unacceptably high, making it the second leading cause of death in children $<5$ years [14]. The factors associated include younger age, low socioeconomic status, poor maternal education, low birth weight, malnutrition, and poor sanitation and hygiene practices [15].

\section{Picky eating}

Picky eating is a complex behavior commonly seen in children which can significantly affect their growth, cause impaired cognition and poor immunity. The findings of the cross-sectional study from Telangana on 1652 parents of preschool children in the age group of $1-6$ years revealed that $58.9 \%$ of the children were picky eaters [16].

\section{Triple burden of malnutrition}

Rising number of Indian children and adolescents experiencing triple burden of malnutrition is a public health concern [17]. Triple burden of malnutrition includes undernutrition (stunting,

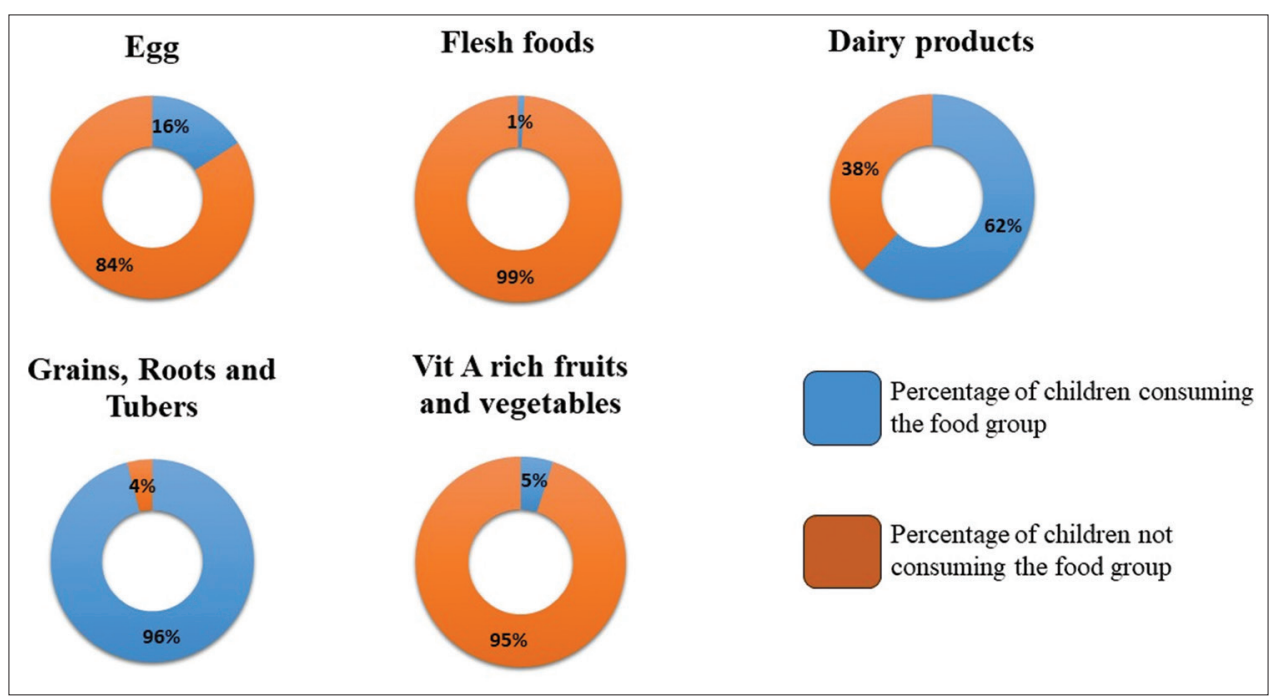

Figure 1: Percentage consumption of different food groups 
wasting), overnutrition and micronutrient deficiency. The CNNS 2016-2018 report mentioned that $2 \%$ of children $<5$ years of age were overweight or obese. The mean wasting (WHZ) score for the population was -1.0 , the prevalence of overweight or obese was significantly higher than expected and could therefore indicate an upsurge of overweight and obesity in the country [10]. In 2020, the National Family Health Survey (NFHS) 5 (20192020 ) reported significantly increased prevalence of overweight in children of age group $0-59$ months from $0.7 \%$ (2015-2016) to $3.4 \%$ (2019-2020) in Telangana and 2.1\% (2015-2016) to 4.3\% (2019-2020) in West Bengal [2].

Prevalence of undernutrition in 0-4 years age group, in India, was as follows [18]:

- Stunted (HAZ $<-2$ SD)- $35 \%(<5$ yr.) $/ 1$ in 3 children is stunted

- Wasted (WHZ $<-2 \mathrm{SD}$ )- $17 \%$ ( $<5 \mathrm{yr}$ ) $/ 1$ in 5 children has low weight for age

- Underweight (WAZ $<-2$ SD)- $33 \%$ ( $<5$ yr. $) / 1$ in 3 children is underweight.

NFHS 5 (2019-2020) reported the rising prevalence of undernutrition in Telangana. There has been a significant rise in stunting, wasting and underweight in under 5 years children. Every fifth kid in Telangana, West Bengal, Assam, Karnataka, Nagaland, Goa, and Jammu and Kashmir was found to be underweight [2].

Hence, there is a dire need to optimize the nutritional intake in preschoolers to prevent both the spectrums of malnutrition. Therefore, through this round table opinion, we aim to collect expert opinions from practicing pediatricians on the nutritional concerns, feeding behaviors and existing problem areas in this age group and how this nutritional gap can be addressed.

\section{METHODS}

About 28 practicing pediatricians were selected from Telangana and West Bengal purposively, who met the inclusion criteria: pediatricians $>10$ years of experience, interested in preschool nutrition, academic and research oriented and willing to share honest feedback. The selected pediatricians formed the Advisory board. Further the advisory board meetings were conducted on $28^{\text {th }}$ August 2021 virtually in Telangana $(n=12)$ and on $4^{\text {th }}$ September 2021 physically in West Bengal $(n=16)$ with an aim to understand the nutritional concerns of the mothers of preschoolers, to identify the nutritional gaps and ascertain the solutions to improve their nutritional status. The Advisory board meeting was initiated with a brief presentation on preschool nutrition: an Indian perspective. This presentation was based on the preschooler personality, factors affecting growth and development of preschooler, food and snacking patterns of preschoolers, food-based patterns in Indian preschoolers and common problems and nutrition concerns of preschoolers. Thereafter, a focused group discussion was conducted, in which the group of experts put forth their opinions on each topic, this was guided by an expert moderator who introduced the topics, lead and guided the discussion using a set of predetermined questions designed based on the following five key, pre-identified areas of nutrition among preschoolers: (1) Maternal concerns and feeding problems during preschool years, (2) Healthy food drinks, (3) Sugar and fat intake in preschoolers, (4) Importance of prebiotics and DHA in preschool nutrition, (5) Role of doctors in nutrition counseling.

Different sets of questions were used such as probing, follow-up and exit questions to execute a well- rounded discussion on each topic by the moderator. A power point presentation was used as the tool during these discussions. The discussions, insights, and/or opinions of the experts for each of these topics were recorded by the investigator as the minutes of meeting, and an advisory board summary was documented. The opinions obtained from these meetings have been presented under the results and discussion section.

\section{RESULTS AND DISCUSSION}

The key points discussed by the experts as seen in their clinical practise, along with scientific evidence supporting the discussion, were compiled together to arrive at the round table opinion on preschool nutrition in India. The expert opinions from the discussions are presented below:

\section{Maternal Concerns and Feeding Problems in Preschoolers}

Mothers are concerned about their child's nutrition, growth and development. A recent study on mothers of 5 -year-olds reported that majority of the mothers $(60 \%)$ had concerns about their child's eating habit and weight. They resorted to control feeding practises such as restrictive feeding in case of overweight child and lower levels of co-participation in physical activity, and higher levels of using media to control child behavior [19]. The findings from a recent 2019 cross-sectional survey in an outpatient department of tertiary care in Mumbai involving 200 parents of 1-5-year-old children, reported picky eating as a major parental concern, the estimated prevalence rate was $25 \%$, maximum $38 \%$ at $49-60$ months. In picky eaters, $26 \%$ of children were severely stunted, while $38 \%$ were severely wasted as compared to only $4 \%$ in non-picky eaters [20]. In 2-7 years age group, the common feeding problems faced by parents were desirable and undesirable mealtime behaviors, low vegetable consumption, poor appetite, food neophobia [21].

\section{Brief summary of experts' opinions}

In case of mild nutritional deficiencies, specific nutrient rich foods are advised. For example, in vitamin A deficiency, fruits, vegetables, milk, etc. Common nutrient deficiencies observed in preschoolers are iron, Vitamine A, D etc. There is a need for parental counselling to include all food groups in the child's diet. In situations where there is presence of common nutritional deficiencies with/without malnutrition, feeding issues such as 
picky eating, nutritional needs may not be met via diet alone. Here, age specific supplementation can be considered to bridge the nutrition gap.

The December 2016 consensus statement from Infant and Young Child Feeding Chapter of Indian Academy of Pediatrics, suggested scaling up of existing iron, Vitamin A, zinc supplementation and universal salt iodization programs, especially in the vulnerable groups of mothers and children $<5$ years, and also indicated an urgent need for universal Vitamin D supplementation, Vitamin B12 deficiency screening and supplementation and availability of appropriate fortified foods. This indicates that diet alone cannot suffice the nutrient needs and supplementation of specific problem nutrients is needed to prevent micronutrient deficiencies and undernutrition [22].

\section{Health Food Drinks}

Health food drinks (HFD) are specially designed to complement home-made foods and to support height gain, cognition, and overall health in children. NHANES 2015-2016 survey revealed that close to half $(44 \%)$ of $2-5$-year-olds consumed a sugarsweetened beverage daily [23]. Hence, it is important to introduce healthy beverages to young children to meet the nutritional needs and support growth and development [22]. A review from Cochrane database by Das et al. (2019) shortlisted 43 selected studies, 19585 participants (17,878 children), which revealed the effects of food fortification with multiple micronutrients. Positive outcomes were seen in reducing anemia, micronutrient deficiencies and malnutrition in children [24].

\section{Brief summary of experts' opinions}

Nutritional supplements/powders designed for preschoolers are fortified with micronutrients of importance during this growing phase of life. They also have nutrients which are commonly found to be deficient in these children. Hence, in case of undernutrition, recovering from an illness and micronutrient deficiency it is convenient to advise nutritional supplements to ensure adequate nutrition and required combination of nutrients. Suggesting supplements/powders that are flavored and can be added to milk are more feasible in preschoolers as milk is regular part of their diet. These HFD are available in child friendly flavors coming to a quick rescue for picky eaters. Being in liquid form, these nutrients can be delivered in a simple way during situations of time constraints like early morning school hours or before play. American Academy of Pediatric Dentistry recommended that establishing healthy beverage patterns in the first 5 years can help in promoting healthy eating patterns, meet adequate intake of healthy nutrients while reducing excess consumption of sugars and saturated fats [25].

\section{Sugar and Fat Intake in Preschoolers}

With advancing technology, rapid urbanization and sedentary lifestyle, obesity, and other metabolic diseases are on the rise and children are not an exception to that. Thus, laying correct foundation in nutrition right from early childhood is important. In 2015, India had second highest number of obese children in the world, after China [26]. Encouraging intake of nutritious foods over high sugar and high fat foods is required. With growing economy and busy work lives, parents often resort to quick fixes and ready to eat/ready to make alternatives.

\section{Brief summary of experts'opinions}

In several panellists' opinion, approximately $30 \%$ of children were obese as seen in practise. Excess intake of sugar, sweetened beverages, high fat foods (French fries, chips, burgers and other fried items) should be restricted. Intake of healthy and easy to prepare nutritious alternatives should be imposed. Lower awareness and knowledge for nutrition among the parents, made their children choose more sugar and fat rich foods. While, higher the nutritional knowledge of the mothers better were the eating habits of their children [27]. Thus, educating parents on healthy eating options is important with the help of a healthcare professional.

\section{Importance of Prebiotics and DHA in Preschool Nutrition}

Prebiotics are non-digestible food components that result in specific changes in the composition and/or activity of the gastrointestinal microbiota, thus conferring health benefit(s) on host health. Prebiotics reduce pathogenic bacteria, improve gut health and immune functions, also influence memory and learning [28].

Giving kinder garden children a prebiotic inulin type fructan supplement, during the cold weather, has shown to reduce febrile episodes that require medical attention and helps to lower incidence of sinusitis. Modulation in gut microbiota could be the reason behind these beneficial effects. A randomized controlled trial was conducted on 258 healthy children (3-6 years of age), who consumed $6 \mathrm{~g}$ /day of prebiotic inulin-type fructans or maltodextrin during a 24 weeks intervention and during antibiotic treatment. Higher relative abundance of bifidobacteria was observed in the group given prebiotics [29]. Similar results were found in another study, where supplementation with $6 \mathrm{~g} /$ day fructans or maltodextrin for 24 weeks in children aged 3-6 years, modified the gut microbiota composition (increased relative abundance of Bifidobacterium and Lactobacillus), resulted in softer stools and significantly lower incidence of febrile episodes requiring medical attention [30].

DHA is a structural component of membranes, found specifically in the central nervous system. In relation to brain function, DHA has a significant role in neuronal cell growth, differentiation and neuronal signalling. Most of the data from studies on humans point out to the contribution of DHA to development of optimal visual acuity [31]. DHA consumption is beneficial for brain activity, cognitive function, and improves learning, behavior and school performance in healthy children [32]. 
Brief summary of experts'opinions

Prebiotics: Including prebiotics is beneficial to health due to the following reasons: (1) Help in modulating immunity [33]: Prebiotics enhance immunity by neutralizing and preventing adhesion of pathogens, aid in promoting the growth of commensal flora, outcompeting pathogens, help in enhancing protein transcription for barrier integrity, play a role in SCFA production which lowers the gut $\mathrm{pH}$, favoring growth of beneficial bacteria. (2) Helps to improve nutrient absorption: Studies showed that non-digestible oligosaccharides such as fructo-oligosaccharides (FOS) and inulin can enhance the intestinal mineral absorption by their prebiotic property. Once they reach the colon undigested, they are fermented by the bifidobacteria to produce short chain fatty acids (SCFA). SCFA lowers the cecal PH and increase the mineral concentration in the liquid phase and enhance their absorption [34]. FOS increase calcium uptake and stimulate bifidobacterium growth in the colon, which helps to reduce iron intake by the intestinal pathogens, thus increasing iron absorption. Inulin was seen to increase vitamin $\mathrm{D}$ and $\mathrm{E}$ absorption. Gut bacteria also produce Vitamin B3, B5, B6, B12, Biotin and Vitamin $\mathrm{K}$ [35]. Adding prebiotics to multi nutrient powders (MNP's) help to alleviate the negative effect of supplemental iron to the gut [36]. Studies have also shown that consuming mixture of prebiotic short- and long-chain inulin-type fructans daily increases calcium absorption and enhance bone mineralization during pubertal growth [37].

DHA: Experts agreed on the fact that DHA is an important nutrient in a preschoolers diet as it plays a role in cognitive development of the child.

\section{Role of Doctors in Nutrition Counselling}

A research study (2012) on physician's nutrition counseling practices in Chattisgarh, in India revealed that $52 \%$ physician respondents thought it was futile, $62 \%$ thought it was time consuming, while majority $(76 \%)$ were willing to undergo additional nutrition training [38]. In 2020, consensus statement by Indian Academy of Pediatrics on early childhood included recommendations that pediatricians should lead awareness generation in the care givers, preschool teachers and routinely talk to parents and care givers of children under 5 years of age regarding adequacy of diet, physical activity, sedentary behavior and sleep [39].

\section{Brief summary of experts' opinions}

The doctors need to invest some time in nutritional counseling each time they talk to their patients to improve their nutritional status while addressing the medical concerns, discourage unhealthy behavior such as increased consumption of fried food and sweetened beverages. In addition, they must counsel their patients about nutritional supplements if deficiency is the issue [40].

Provision of educative materials such as leaflets, charts on display or small videos on important nutrients during preschool period with their food sources, importance of healthy eating and exercise can be used to create awareness on a healthy lifestyle and ensure healthy future for children. They can further refer patients to qualified dietitians for appropriate and individualized nutrition guidance [41].

\section{CONCLUSION}

The advisory board members emphasized that the dietary habits during preschool years, determine the physical, social and cognitive development and have an impact on health even in adulthood. Thus, it is important to address the nutritional challenges of preschoolers early on in life. The discussion summed up with the viewpoint that there is a pressing need to encourage adequate nutrient intake to prevent under and over nutrition and bridge the gap in micronutrient intake emphasizing on nutrient absorption as well. Identifying the nutritional concerns, deficiencies and taking corrective action by counseling on the importance of a balanced diet, supplementation wherever required is the need of the hour. Consumption of healthy calories from complex carbohydrates, protein and essential fatty acids is advised and it is desirable to move away from consumption of unhealthy, high fat and high sugar foods. Considering the time constraints and hectic lifestyle of parents, education on easy to prepare and convenient healthy food options is a must during nutrition counseling by health care professionals to ensure intake of a well-balanced diet. This would help in reducing the deficiencies, improving the nutritional status of preschoolers, ensuring them a healthy future and aid in attenuating the surge of under 5 malnutrition prevalence in India.

\section{ACKNOWLEDGEMENT}

We would like to acknowledge the views shared by-

Dr. Apurba Kumar Ghosh, Dr. Sukanata Bhattacharya, Dr. Soumitra Dutta, Dr. Anup Kumar Mangal, Dr. Subhashish Bhattacharya, Dr. Md. Shams Qamar, Dr. Abhijit Sarkar, Dr. Prabir Bhaumik, Dr. Krishnendu Mukherjee, Dr. Asitava Roy Mahapatra, Dr. Saumitra Mandal, Dr. Lokesh Pande, Dr. Nitish Kumar, Dr. Prince Parakh, Dr. Nibir Chakrabarti from West Bengal. Dr. Michael Aranha, Dr. Rama Krishna Prasad, Dr. Narreddy Ramcharan Reddy, Dr. Malluvalasa Simhachalam, Dr. M. Srilata, Dr. Mrudula Gunukula, Dr. P. Sateesh Kumar, Dr. Munnam Venkata Hari Chandra Mouli from Andhra Pradesh and Telangana.

\section{REFERENCES}

1. World Health Organization. Departmental News. Malnutrition is a World Health Crisis. Geneva: World Health Organization; 2019. Available from: https://www.who.int/news/item/26-09-2019-malnutrition-is-a-worldhealth-crisis [Last accessed on 2021 Sep 13].

2. National Family Health Survey-5 2019-20. Ministry of Health and Family Welfare. State Fact Sheets. Institute of Population Sciences; 2020. Available from: http://www.rchiips.org/nfhs/nfhs-5_fcts/nfhs-5\%20state $\% 20$ factsheet $\% 20$ compendium_phase-i.pdf. [Last accessed on 2021 Sep 13].

3. Centre for Disease Control and Prevention. Child development, National Center on Birth Defects and Developmental Disabilities; 2021. Available 
from: https://www.cdc.gov/ncbddd/childdevelopment/positiveparenting/ preschoolers.html [Last accessed on 2021 Feb 22].

4. French S, Lally M. Early childhood. In: Lifespan Development-A Psychological Perspective. $2^{\text {nd }}$ ed., Ch. 4. Martha Lally, Suzanne ValentineFrench Publishers; 2019. p. 115-63.

5. MacNamara D. Rest, Play, Grow: Making Sense of Preschoolers (or Anyone Who Acts Like One). Vancouver: Aona Books; 2016. p. 1201.

6. Pem D. Factors affecting early childhood growth and development: Golden 1000 days. Adv Pract Nurs 2015;1:101.

7. Niemeier BS, Duan YP, Shang BR, Yang J. Parental influences on weightrelated health behaviors in western and eastern cultures. Child Care Health Dev 2017;43:259-66.

8. Ansuya B, Nayak BS, Unnikrishnan B, George A, Shashidhara YN, Mundkur SC, et al. Risk factors for malnutrition among preschool children in rural Karnataka: A case-control study. BMC Public Health 2018;18:283.

9. United Nations Children's Funds. Potential Impact of Sanitation; 2019. Available from: https://www.unicef.org/india/media/1221/file/potentialimpact-of-sanitation.pdf. [Last accessed on 2021 Sep 15].

10. Comprehensive National Nutrition Survey Report (2016-2018), Chapter 4-Infant and Young Child Feeding and Diets, Ministry of Health and Family Welfare (MoHFW), Government of India, UNICEF and Population Council, New Delhi; 2018. p. 71. Available from: https://www.nhm.gov.in/ writereaddata/1892s/1405796031571201348.pdf. [Last accessed on 2021 Sep 15].

11. Priyadarshini $\mathrm{P}$, Gurunathan D. Role of diet in ECC affected South Indian children assessed by the HEI-2005: A pilot study. J Family Med Prim Care 2020;9:985-91.

12. World Health Organization. Anemia Overview. Geneva: World Health Organization; 2021. Available from: https://www.who.int/health-topics/ anaemia\#tab=tab_1. [Last accessed on 2021 Sep 15].

13. Bharti LK, Kumar B. Constipation in Indian children. Clin Surg 2017;2:1644.

14. Paul P. Socio-demographic and environmental factors associated with diarrhoeal disease among children under five in India. BMC Public Health 2020;20:1886.

15. Park K. Epidemiology of communicable disease. In: Textbook of Preventive and Social Medicine. 19 $9^{\text {th }}$ ed. Jabalpur, India: Banarsidas Bhanot; 2007. p. 142-7.

16. Kumar KP, Srikrishna S, Pavan I, Chary E. Prevalence of picky eating behavior and its impact on growth in preschool children. Int J Contemp Pediatr 2018;5:714-9.

17. Nguyen PH, Scott S, Headey D, Singh N, Tran LM, Menon P, et al. The double burden of malnutrition in India: Trends and inequalities (2006-2016). PLoS One 2021;16:e 0247856.

18. Comprehensive National Nutrition Survey Report (2016-2018), Chapter 5-Anthropometric Status of Children and Adolescents, Ministry of Health and Family Welfare (MoHFW), Government of India, UNICEF and Population Council, New Delhi; 2018. p. 101. Available from: https:// www.nhm.gov.in/WriteReadData/1892s/1405796031571201348.pdf. [Last accessed on 2021 Sep 16].

19. Haines J, Downing KL, Tang L, Campbell KJ, Hesketh KD. Associations between maternal concern about child's weight and related behaviours and maternal weight-related parenting practices: A cross-sectional study. Int J Behav Nutr Phys Act 2018;15:104.

20. Shettiwar S, Wade M. Correlates of picky eating behaviour in children and its effect on growth. Int J Contemp Pediatr 2019;6:2444-9.

21. Sdravou K, Fotoulaki M, Emmanouilidou-Fotoulaki E, Andreoulakis E, Makris G, Sotiriadou F, et al. Feeding problems in typically developing young children, a population-based study. Children (Basel) 2021;8:388.

22. Bharadva K, Mishra S, Tiwari S, Yadav B, Deshmukh U, Elizabeth KE, et al. Infant and young child feeding chapter of Indian academy of pediatrics; members of the national consultative meet. Prevention of micronutrient deficiencies in young children: Consensus statement from infant and young child feeding chapter of Indian academy of pediatrics. Indian Pediatr 2019;56:577-86.

23. Moshfegh AJ, Garceau AO, Parker EA, Clemens JC. Beverage Choices among Children: What We Eat in America, NHANES 20152016. Food Surveys Research Group Data Brief No. 22; 2019. Available from: https://www.ars.usda.gov/arsuserfiles/80400530/pdf/ dbrief/22_beverage_choices_children_1516.pdf. [Last accessed on 2021 Sep 16].

24. Das JK, Salam RA, Mahmood SB, Moin A, Kumar R, Mukhtar K, et al. Food fortification with multiple micronutrients: Impact on health outcomes in general population. Cochrane Database Syst Rev 2019;12:CD011400.

25. American Academy of Pediatric Dentistry. Healthy Beverage Consumption in Early Childhood: Recommendations from Key National Health and Nutrition Organizations: Summary of Oral Health Considerations. The Reference Manual of Pediatric Dentistry. Chicago, Ill.: American Academy of Pediatric Dentistry; 2020. p. 547-50.

26. GBD 2015 Obesity Collaborators, Afshin A, Forouzanfar MH, Reitsma MB, Sur P, Estep K, et al. Health effects of overweight and obesity in 195 countries over 25 years. N Engl J Med 2017;377:13-27.

27. Lee $\mathrm{Y}$, Joo $\mathrm{N}$. The awareness level and needs for education on reducing sugar consumption among mothers with preschool children. Nutr Res Pract 2016;10:229-36.

28. Davani-Davari D, Negahdaripour M, Karimzadeh I, Seifan M, Mohkam M, Masoumi SJ, et al. Prebiotics: Definition, types, sources, mechanisms, and clinical applications. Foods 2019;8:92.

29. Soldi S, Vasileiadis S, Lohner S, Uggeri F, Puglisi E, Molinari P, et al. Prebiotic supplementation over a cold season and during antibiotic treatment specifically modulates the gut microbiota composition of 3-6 year-old children. Benef Microbes 2019;10:253-63.

30. Lohner S, Jakobik V, Mihályi K, Soldi S, Vasileiadis S, Theis S, et al. Inulintype fructan supplementation of 3-to 6-year-old children is associated with higher fecal bifidobacterium concentrations and fewer febrile episodes requiring medical attention. J Nutr 2018;148:1300-8.

31. Lauritzen L, Brambilla P, Mazzocchi A, Harsløf LB, Ciappolino V, Agostoni C. DHA effects in brain development and function. Nutrients 2016;8:6.

32. Kuratko CN, Barrett EC, Nelson EB, Salem N Jr. The relationship of docosahexaenoic acid (DHA) with learning and behavior in healthy children: A review. Nutrients 2013;5:2777-810.

33. McKeen S, Young W, Mullaney J, Fraser K, McNabb WC, Roy NC. Infant complementary feeding of prebiotics for the microbiome and immunity. Nutrients 2019;11:364.

34. Yap KW, Mohamed S, Yazid AM, Maznah I, Meyer DM. Dose-response effects of inulin on the faecal short-chain fatty acids content and mineral absorption of formula-fed infants. Nutr Food Sci 2005;35:208-19.

35. Costa G, Vasconcelos Q, Abreu G, Albuquerque A, Vilarejo J, Aragão G. Changes in nutrient absorption in children and adolescents caused by fructans, especially fructooligosaccharides and inulin. Arch Pediatr 2020;27:166-9.

36. Paganini D, Zimmermann MB. The effects of iron fortification and supplementation on the gut microbiome and diarrhea in infants and children: A review. Am J Clin Nutr 2017;106 Suppl 6:1688S-93S.

37. Abrams SA, Griffin IJ, Hawthorne KM, Liang L, Gunn SK, Darlington G, et al. A combination of prebiotic short-and long-chain inulin-type fructans enhances calcium absorption and bone mineralization in young adolescents. Am J Clin Nutr 2005;82:471-6.

38. Sharma A, Pati S, Chakravarty N, Chauhan AS. Physicians' nutritional counselling practices: A study in district hospitals of Chhattisgarh. Int J Med Public Health 2013;3:103-6.

39. Bharadva K, Shastri D, Gaonkar N, Thakre R, Mondkar J, Nanavati R, et al. Consensus statement of Indian academy of pediatrics on early childhood development. Indian Pediatr 2020;57:834-41.

40. Truswell AS, Hiddink GJ, Blom J. Nutrition guidance by family doctors in a changing world: Problems, opportunities, and future possibilities. Am J Clin Nutr 2003;77 Suppl 4:1089S-92S.

41. Durkin M. Nutrition. American College of Physicians Internist; 2017. Available from: https://www.acpinternist.org/archives/2017/01/nutritioncounseling.htm. [Last accessed on 2021 Sep 16].

Funding: None; Conflicts of Interest: None Stated.

How to cite this article: Rahul P, Khandeparkar A. Perspectives in preschool nutrition. Indian J Child Health. 2021; 8(11):380-385. 